\title{
Diffuse cystic lung disease due to pulmonary metastasis of bronchogenic adenocarcinoma
}

\author{
Tyler Overman BS, Abdurahim Aloud MD
}

\section{CASE}

A 75-year-old woman who has never smoked presented to the clinic with a chronic daily cough. The cough was dry and occasionally productive of white phlegm; it was associated with mild dyspnea and an unintentional weight loss of 12 pounds over six months. She denied fever, chills, chest pain, and night sweats. She had no history of any exposure to tuberculosis or prior malignancy and had a negative PPD test.

Initial chest $\mathrm{x}$-ray showed numerous rounded cavitary $1 \mathrm{~cm}$ nodules throughout both lungs mostly at the bases (Figure 1). Computed tomography of the chest showed "too numerous to count" pulmonary nodules, including cavitary nodules, focal consolidation in the left lower lobe, and few slightly enlarged hilar and mediastinal nodes (Figure 2). The differential diagnosis at the time included metastasis and infection.

A bronchoscopy with BAL and transbronchial lung biopsy was performed. Viral, fungal, bacterial, and mycobacterial cultures were negative. The initial microscopic examination of the biopsy showed papillary adenocarcinoma; the tumor cells stained positive for the immunohistochemical stains TTF1, napsin, and CK7. The biopsy specimen tested negative for CK20, CDX2, and PAX8 stains. This staining pattern was consistent with adenocarcinoma of primary pulmonary origin. The tumor cells tested positive for EGFR activation mutation associated with response to EGFR tyrosine kinase inhibitors. The tumor cells tested negative for ALK and ROS1 genes.

Computed tomography scans of the abdomen and pelvis, thyroid ultrasound, and tumor markers were

Corresponding author: Abdurahim Aloud Contact Information: Abdurahim.aloud@ttuhsc.edu DOI: 10.12746/swrccc.v6i23.467

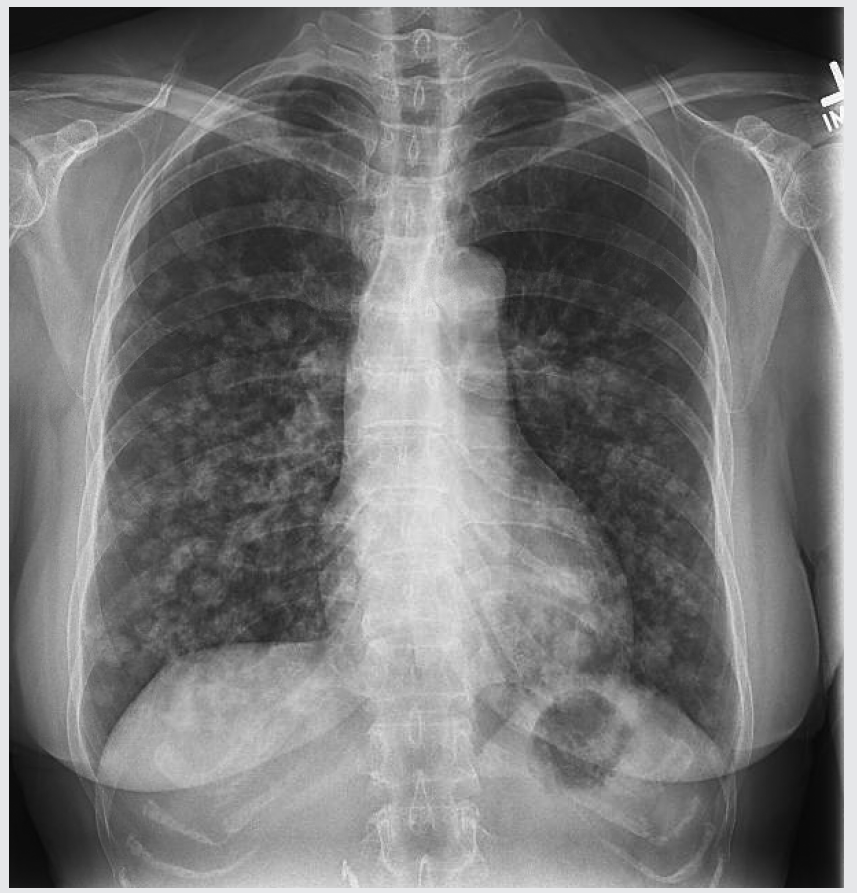

Figure 1. Frontal radiograph of the chest shows innumerable pulmonary nodules throughout the lungs, many of which show cavitation.

ordered. The positive results included an elevated CA 19-9 and CEA; the thyroid ultrasound showed a benign-appearing $6 \mathrm{~mm}$ solid nodule in the inferior aspect of the left lobe and fibronodular changes throughout the thyroid gland consistent with chronic thyroiditis.

\section{Discussion}

A lung cavity is defined as "a gas-filled space", seen as lucency or low-attenuation area, within a pulmonary consolidation, a mass, or a nodule. The cavity wall thickness can vary considerably. At their 

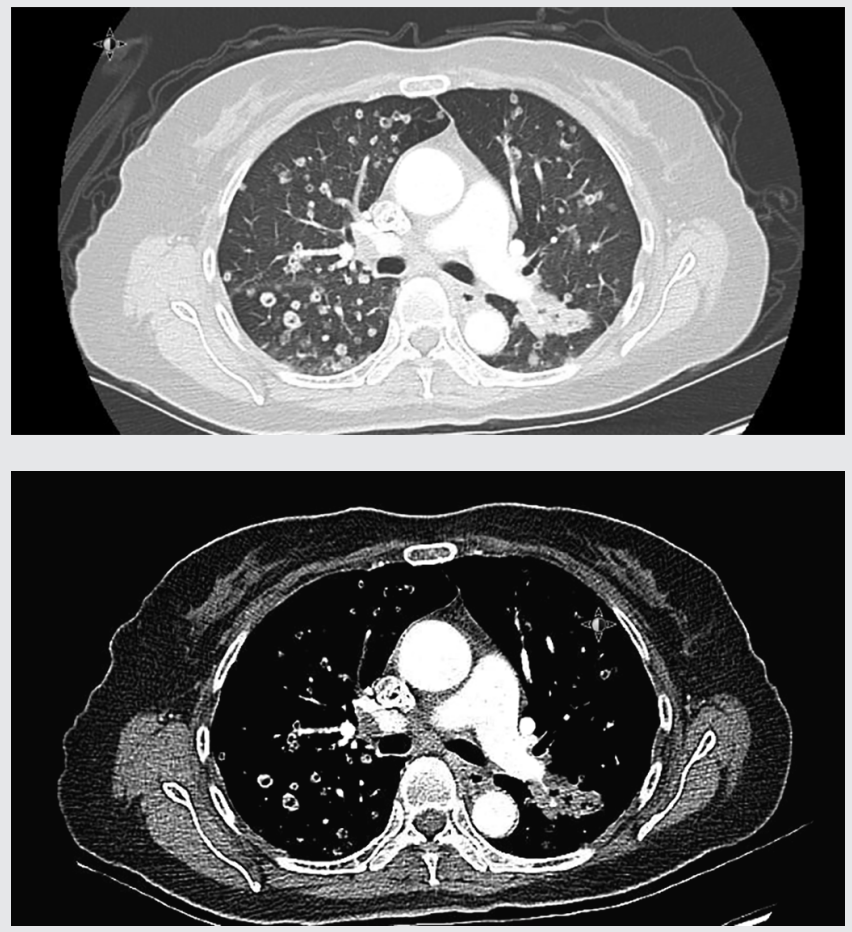

Figure 2. Contrast axial CT images with lung and mediastinal windows shows numerous nodules throughout the lungs, a few of which show cavitation, a patchy opacity in the superior segment left lower lobe and mild right hilar and subcarinal lymphadenopathy.

end-stage presentation, some cavitary diseases may have thin walled cavities or cysts and have a continuous transition from cavities to cysts. A cyst in the lung is defined as any round circumscribed space that is surrounded by an epithelial or fibrous wall of variable thickness, usually thin walled $(<2 \mathrm{~mm})$. It is important to distinguish a cyst from a cavity; cavities are relatively common in pulmonary cancers.

Diffuse cystic lung diseases (DCLD) are a group of disorders characterized by the presence of multiple cysts or cavitary nodules in both lungs. The differential diagnosis includes lymphangioleiomyomatosis (LAM), pulmonary Langerhans cell histiocytosis (PLCH), lymphocytic interstitial pneumonia (LIP), pulmonary amyloidosis, infections, including Pneumocystis jiroveci pneumonia, aspergilosis, septic emboli, Streptococcus pneumoniae, Staphylococcus aureus, Klebsiella pneumoniae, miliary tuberculosis, nontuberculous mycobacteria, and metastatic malignancy, including colorectal adenocarcinoma, lung adenocarcinoma, metastatic leiomyoma, and endometrial stromal sarcoma. Wall thickness and clinical information are important criteria to differentiate between cavitary and cystic lung disease. The differential diagnosis in patients with lung cysts is large. An important distinction is whether the cysts are focal or multifocal and scattered diffusely throughout both lungs, as in DCLD. In the latter case the differential diagnosis usually includes two rare entities, LAM and PLCH. Infrequently DCLD can develop as a result of metastatic malignancies, such as sarcoma, mesenchymal tumors, and colorectal or bronchogenic adenocarcinoma.

Our patient had never smoked and was generally healthy, and the diagnosis of lung cancer and PLCH seemed unlikely. Thyroid papillary adenocarcinoma was initially considered because the initial histopathological examination showed papillary adenocarcinoma, and the thyroid ultrasound showed a benign appearing $6 \mathrm{~mm}$ solid nodule. However, immunohistochemical staining of the tumor cells was positive for TTF1, napsin, and CK7, and was negative for CK20, CDX2, and PAX8. This staining pattern is consistent with pulmonary adenocarcinoma. Consequently, these images represent a rare presentation of metastatic bronchogenic adenocarcinoma.

Napsin $A$ is a cytoplasmic marker identified in type II pneumocytes and alveolar macrophages and is positive in $83 \%$ of lung adenocarcinomas (ADC). It was negative in all squamous cell carcinomas in one study. In a study by Kim evaluating napsin A and TTF-1 staining of pulmonary ADC, napsin A had a positivity rate of $83 \%(44 / 53)$ compared to TTF-1 with a positivity rate of $57 \%(30 / 53)$. In addition, all non-pulmonary ADCs were negative for napsin $A$ and TTF-1. As these studies show, TTF-1 and napsin A are both sensitive and specific for pulmonary ADC. However, napsin A recently has been reported as expressed in renal cell carcinoma, especially the papillary and clear cell types.

Keywords: cystic nodules, metastatic adenocarcinoma, bronchogenic carcinoma, napsin A 
From: The Department of Internal Medicine at Texas Tech University Health Sciences Center in Lubbock, TX Submitted: $3 / 14 / 2018$

Accepted: 4/10/2018

Reviewer: Eman Attaya MD

Conflicts of interest: none

This work is licensed under a Creative Commons

Attribution-ShareAlike 4.0 International License.

\section{REFERENCES}

1. Parkar AP, Kandiah P. Differential diagnosis of cavitary lung lesions. J Belgian Society of Radiology 2016;100(1):100. DOI: http://doi.org/10.5334/jbr-btr.1202

2. Fielli M, Avila F. Diffuse cystic lung disease due to pulmonary metastasis of colorectal carcinoma. Respiratory Medicine Case Reports 2016;17:83-85. doi:10.1016/j.rmcr.2015.12.006.

3. Siddiqui MT. TTF-1 and Napsin a double staining in diagnosing lung adenocarcinoma. J Cytol Histol 2012;3:e103. doi:10.4172/2157-7099.1000e103 